\title{
Efeitos da prática de caminhada de idosos em grupo: un olhar do protagonista
}

\author{
|Effeets of practice of group walk in elderly: a protagonist's look
}

\begin{abstract}
Abstraet
O envelhecimento humano é natural e acompanhado de alterações físicas, mentais e sociais, frequentemente acrescido de doenças crônicodegenerativas. Assim, o cuidar em estratégias grupais que visem à manutenção da funcionalidade é fundamental. O presente estudo de abordagem qualitativa, fenomenológico, não experimental, de caráter descritivo e explicativo, objetivou conhecer a óptica de idosos, conviventes com as patologias crônico-degenerativas, acerca dos efeitos da prática da caminhada em grupo, na Estratégia Saúde da Família do bairro Mangueira, da cidade de Grajaú - MA. Inferiu-se, que, essa prática representa suporte para as necessidades físicas, mentais e sociais dos pesquisados. Esses resultados possibilitam o planejamento e execução de ações efetivas para o sucesso no cuidado ao idoso, mantendo-o funcional, condição crucial para o envelhecimento ativo e saudável, o qual tornará a velhice uma conquista social.
\end{abstract}

\section{Resumo}

The natural and human aging is accompanied by physical changes, mental and social, often plus chronic degenerative diseases. Thus, the care group strategies aimed at maintaining the functionality is key. The present study qualitative approach, phenomenological, non-experimental and descriptive and explanatory, aimed to know the optics of the elderly, living together with the conditions chronic degenerative, about the effects of the practice of walking in a group, the Family Health Strategy in the neighborhood hose, the city of Grajaú, MA. Inferred that this practice is to support the physical, mental and social search. These results enable planning and implementing effective strategies for success in caring for the elderly, keeping it functional key condition for active and healthy aging, which will make old age a social achievement.

\section{Ayslan Gonţalves Torres ${ }^{1}$ Helena Maria Pereira da Silva ${ }^{1}$ Deiane Ferreira do Naseimento Torres ${ }^{1}$ Wellyson da Cunha Araújo Firmo ${ }^{1}$ \\ Arlane Silva Chaves ${ }^{1}$}

Deseritores: Exereicio fisico. Idosos. Patologia.

Keywords: Exereise. Aged. Pathology.

\begin{abstract}
${ }^{1}$ Bacharel em Enfermagem pela Universidade Estadual do Maranhão [UEMA]. Especialista em Doeêneia do Ensino Superior pela Faculdade de Ciêneias, Educação o Teologia do Norte do Brasil [FACETEN]. Atualmente é professor da Faeuldade de Educação de Bacabal [FEBAC] - Departamento de Farmácia e Enfermagem e Professor da UEMA
\end{abstract}

${ }^{2}$ Bacharel em Enfermagem pela Universidade Estadual do Maranhão/Centro de Estudos Superiores de Grajaú [UEMA/CESERA], Especialista em Gestão em Saúde Pública, Saúde da Familia e Coletiva pela Faculdade Adelmar Rosado em parceiria com Instituto Nordeste de Eduạão Superior e Pós-graduạ̣ão [INESPO]

${ }^{3}$ Bacharel em Enfermagem pela Universidade Estadual do Maranhão/Centro de Estudos Superiores de Grajaú [UEMA/CESERA], Especialista em Gestão em Saúde Públicea, Saúde da Familia e Coletiva pela Faculdade Adelmar Rosado em parceria com Instituto Nordeste de Eduacão Superior e Pós-graduacão [INESPO] Professora do departamento de enfermagem da Universidade Estadual do Maranhão [UEMA/CESGRA]

${ }^{4}$ Graduado em Farmácia pela Faculdade de Imperatriz [FACIMP], Especialista em Saúde Pública pelo Instituto Nordeste de Educação Superior e Pós-graduaţão [INESPO]. Pós-graduando em Farmacologia pela Universidade Católiea Dom Boseo [UCDB] e Mestrando em Saíde e Ambiente pela Universidade Federal do Maranhão [UFMA]. Professor da Faculdade de Educação de Bacabal [FEBAC]

${ }^{5}$ Bacharel em Enfermagem pela Universidade Estadual do Maranhão/Centro de Estudos Superiores de Grajaú [UEMA/CESERA], Especialista em Gestão em Saúde Públiea, Saúde da Familia e Coletiva pela Faculdade Adelmar Rosado em parceria com Instituto Nordeste de Eduatãa Superior e Pós-uraduatão [INESPO]. Professora do departamento de enfermagem da Universidade Estadual do Maranhão [UEMA/CESERA] \& do Departamento de Enfermagem da Universidade Federal do Maranhão [UFMA] Campus - Imperatriz

Para correspondência:

Ayslan Gonçalves Torres

email: ayslan_goncalves@hotmail.com 
Introdựão

O envelhecimento é um processo natural e progressivo, que caracteriza uma etapa da vida do homem e dá-se por diversas alterações físicas, mentais e sociais, decorrentes do desgaste das estruturas corporais, psíquicas e cognitivas. Para o indivíduo idoso essa fase representa a ponderação sobre a própria existência, pois este conclui que alcançou muitos objetivos, mas também sofreu muitas perdas, das quais a saúde é vista como a que mais ocasionou alterações ${ }^{1}$.

A Organização Pan-Americana de Saúde (OPAS) define o envelhecimento como um processo sequencial, individual, acumulativo, irreversível, universal, não patológico. Geralmente ocorre a deterioração de um organismo, comum a todos os membros de uma espécie, de maneira que o tempo o torne cada vez menos capaz de fazer frente ao estresse do meio ambiente e, portanto, aumente sua possibilidade de morrer ${ }^{2}$.

Já o Ministério da Saúde define o envelhecimento populacional como a mudança na estrutura etária da população, com um aumento do número relativo das pessoas acima de determinada idade, considerada como definidora do início da velhice. No Brasil, é considerado como idosa a pessoa que tem 60 anos ou mais de idade ${ }^{3}$.

A sociedade ao longo do tempo sofre significativas modificações e a transição demográfica é uma das mais evidenciadas atualmente. Mendes et $a l^{1}$, dizem que o envelhecimento da população é um fenômeno mundial, que teve início nos países desenvolvidos, em decorrência da queda de mortalidade. Acrescentado às grandes conquistas do conhecimento médico, urbanização adequada das cidades, melhoria nutricional, elevação dos níveis de higiene pessoal e ambiental tanto em residências como no trabalho, assim como, em decorrência dos avanços tecnológicos. Estes fatores ficaram evidenciados no final da década de 40 e início dos anos 50 .

Segundo Benedetti et $a l^{4}$, baseados em relatório da Organização das Nações Unidas (ONU), em 2005 o percentual mundial de pessoas acima de 60 anos era $11,7 \%$. Entre os países que apresentam percentual de idosos maior que 25\% estão: Japão, Itália, Alemanha, Suécia, Portugal, França, Suíça, Grécia e Espanha entre outros que permanecem nesse percentual. O Brasil aparece na marca dos $10 \%$.

De acordo com o Instituto Brasileiro de Geografia e Estatística (IBGE), atualmente há no
Brasil, aproximadamente, 20 milhões de pessoas com idade igual ou maior do que 60 anos. Segundo projeções estatísticas da Organização Mundial de Saúde - OMS, no período de 1950 a 2025, o grupo de idosos no país deverá ter aumentado em quinze vezes, enquanto a população total em cinco. Assim, o Brasil ocupará o sexto lugar quanto ao contingente de idosos no mundo, alcançando, em 2025, cerca de 32 milhões de pessoas com 60 anos ou mais de idade ${ }^{3}$.

Hoje é notória e louvável a preocupação que se manifesta para com o envelhecimento da população, por meio do desenvolvimento de ações e políticas voltadas para o benefício da pessoa idosa. Entretanto não é fato a realidade desejada.

É preciso por em prática os direitos determinados pela Lei $\mathrm{n}^{\circ} 8.080$, do Sistema Único de Saúde (SUS), pela Política Nacional de Saúde da Pessoa Idosa (PNSPI), pelo Estatuto do Idoso, pelo Pacto pela Saúde e pelas portarias e resoluções decorrentes 5 . O que certamente servirá de suporte para sanar ou ao menos minimizar os efeitos negativos do envelhecimento, senão fazê-lo, dentro das limitações existentes, positivo para o sujeito que envelhece e para a sociedade em geral.

Uma forma de se contribuir para isso, pode ser por meio de políticas públicas que incentivem a prática de atividade física, pois o exercício implica na repetição de determinado movimento. Assim, dizemos que estamos nos exercitando quando realizamos movimentos de modo encadeado e sequenciado. Quando se associa o movimento à saúde, geralmente, ele é compreendido como sinônimo de atividade física e saúde, ainda que sejam definições distintas. Sendo assim, infere-se que o praticante de atividade física com acompanhamento está associado à prevenção de doenças ${ }^{6}$.

Nesse contexto, o profissional de enfermagem tem espaço para dar contribuição para o envelhecimento ativo e saudável, por meio da implantação de estratégia de atividade física orientada como grupo de caminhada, pois de acordo com Gonçalves et $a l^{7}$, está associada à prevenção de doenças, manutenção do corpo forte e resistente. Podemos elencar, ainda, as melhorias nas relações sociais devido à convivência em grupo, propiciadas pelo contato com outras pessoas de mesma idade durante o envolvimento com a prática, assumindo peculiar importância na contribuição para a qualidade de vida da pessoa idosa.

Essa é uma estratégia defendida por 
Freitas et al ${ }^{8}$ ao afirmarem que $90 \%$ das pessoas que praticam exercício físico, preferem fazê-lo com um companheiro ou com um grupo a se exercitarem sozinhos.

Santana e Santos $^{9}$ apontam que oferecer espaços de sociabilização e de interação no processo de cuidar, propicia ambiente favorável à estimulação do imaginário, à revelação de medos escondidos e de coisas esquecidas e recalcadas pelo idoso, como forma de eliminar ou diminuir o mal-estar.

Além disso, o Ministério da Saúde lista os principais benefícios biológicos, psicológicos e sociais proporcionados por essa prática: melhor funcionamento corporal, diminuindo as perdas funcionais, favorecendo a preservação da independência; redução no risco de morte por doenças cardiovasculares; controle da pressão arterial; manutenção da densidade mineral óssea, com ossos e articulações mais saudáveis; melhora a postura e o equilíbrio. Além de favorecer o controle do peso corporal; melhora o perfil lipídico e aumenta a utilização da glicose pelas células; regula a função intestinal; diminuição de quadros álgicos; aumenta a resposta imunológica; mais qualidade do sono; ampliação do contato social; correlações favoráveis com redução do tabagismo e abuso de álcool e drogas; e diminuição da ansiedade, do estresse, melhora do estado de humor e da autoestima ${ }^{10}$.

Entendendo a subjetividade do cliente como fator fundamental para o cuidado de qualidade, fez-se necessário investigá-la de forma holística. Nessa perspectiva, conhecer o que pensa o sujeito, acerca dos resultados dos cuidados que lhe são prestados, far-se-á num estimável complemento para o progresso da eficácia da assistência à saúde do idoso na ESF.

A experiência da prática da caminhada de idosos em grupo é feita em uma ESF da cidade de Grajaú - MA. Seus resultados são passíveis de mensuração que segundo a equipe prestadora de assistência, demonstram modificações significativamente positivas nas condições clínicas de idosos que convivem com doenças crônicodegenerativas, como hipertensão e diabetes.

Estes são resultados plausíveis, no entanto, os aspectos físicos de dados objetivos não são suficientes para a compreensão de forma holística dos efeitos da prática em questão. Viu-se, portanto, a necessidade de realizar o presente estudo, objetivando conhecer a óptica dos idosos acerca dos efeitos da prática da caminhada em grupo, buscando identificar a contribuição dessa atividade para o envelhecimento ativo e saudável.

\section{Metodologja}

Trata-se de uma pesquisa de campo qualitativa, um estudo fenomenológico, não experimental, que buscou descrever, compreender, interpretar e explicar os fenômenos que se apresentam à percepção dos idosos que praticam caminhada em grupo.

O estudo foi desenvolvido na cidade de Grajaú - MA, no período de 28 de abril a 29 de maio de 2010, por meio da aplicação de um roteiro de entrevista semiestruturado a todos 10 os idosos matriculados no grupo de caminhada da ESF Mangueira.

O grupo que é composto atualmente por 10 idosos, que estão cadastrados na referida ESF por alguma doença crônico-degenerativa, foi formado pelo enfermeiro responsável da equipe de saúde da família do bairro Mangueira no município de Grajaú - MA no ano de 2007.

$O$ critério de inclusão na pesquisa consistiu nos idosos que frequentam assiduamente as atividades em um período de cerca de um ano e três meses a três anos, convivem com patologia crônico-degenerativa, e se dispuseram a participar voluntariamente da pesquisa, através do Termo de Consentimento Livre e Esclarecido. Enquanto o critério de exclusão foi justamente os que não contemplaram o critério de inclusão.

A maioria dos idosos que participaram do estudo eram viúvos, todos aposentados sem nenhuma atividade laboral, residentes no município de Grajáu - MA, convivendo com doenças como o diabetes ou hipertensão arterial.

Destaca-se que este estudo atendeu às exigências do Conselho Nacional de Saúde, obteve aprovação do Comitê de Ética em Pesquisa da Faculdade NOVAFAPI sob o processo CAAE $n^{\circ}$ 0070.0.043.000-10, em 20 de abril de 2010, atendendo à Portaria n ${ }^{\circ} 196 / 96$ do Conselho Nacional de Saúde que normatiza as pesquisas envolvendo seres humanos. A identificação de cada participante foi pela letra "I" de idoso e enumerada conforme a ordem de coleta dos dados.

\section{Resultados e Diseussão}

Os dez idosos entrevistados encontram-se na faixa etária de 62 a 76 anos de idade, 7 (sete) deles são do sexo feminino e 3 (três) do sexo masculino. Metade é casada, um solteiro e os demais viúvos. Quanto à escolaridade, a maior 
parte cursou o ensino fundamental incompleto, apenas 1 (um) o ensino fundamental completo, 01(um) o ensino médio incompleto e 4 (quatro) não são alfabetizados. $\mathrm{O}$ tempo de participação no grupo de caminhada varia de um ano e três meses a três anos. Enquanto as doenças crônico-degenerativas citadas pelos participantes incluem Hipertensão Arterial (HA), Diabetes Mellitus (DM), Osteoporose, Artrose, Bursite e ainda Câncer de pele, Asma e Hipercolesterolemia.

Assim, um dos motivos elencados pelos entrevistados quanto à participação no grupo de caminhada, foi à necessidade de cuidados de saúde e prevenção de complicações de suas patologias como a crise hipertensiva. Além disso, a constante insatisfação com o peso, dores e depressão, bem como a oportunidade de socialização, entretenimento, influência e suporte de amigos e cônjuge. Estes foram os fatores determinantes para o ingresso dos idosos no grupo de caminhada.

Observemos as falas a seguir:

"Mulher, foi sobre a pressão que alterou, aí eu, eu digo é obrigado eu ir lá, (risos..), aí eu falei para (ACS) e ela disse vai menina, tu vai, aí um dia eu cheguei lá, aí estavam, aí eu falei gente vim medir a pressão que ela está meio assulerada, estava 22..." (I1)

"[...] e olha, eu estava pesando 69 quilo aí eu estava cansada, eu digo, não, eu vou fazer essa caminhada que o povo diz."

"É... o motivo é saúde né? porque eu vivia muito cheia de dor [...]" (I4)

"Rapaz, o motivo é o seguinte: que é uma diversão para os idosos, pra mim e outros idosos, é uma diversão né, tem uma doutora lá pra gente fazer as fisio né, tem aquela animação lá, vez em quanto aparece uma brincadeirinha pra gente, então é uma diversão pra mim e pra outros idosos também né? é bom, pra mim eu acho bom aquela diversão alí das caminhada né?" (I5)

"O motivo foi porque eu vivia doente e a técnica de enfermagem e o enfermeiro vieram aqui e me levaram prá lá." (I10)

Corroborando com o exposto acima, Freitas et $a l^{8}$ define motivo como alguma força interior, impulso e intenção, que leva uma pessoa a fazer algo ou agir de certa forma. Assim, qualquer discussão sobre motivação implica investigar os motivos que influenciam um determinado comportamento, ou seja, todo comportamento é motivado, é impulsionado por motivos.

O grupo atua na prevenção secundária da saúde dessas pessoas, as quais já se encontram acometidas por patologias como as doenças crônico-degenerativas, particularmente comuns em indivíduos de sobrevida avançada nos países em desenvolvimento.

Nessa fase da vida, reforçam Bessa e Silva11, eles decidem por livre vontade ou por influência de outras pessoas a procurarem um grupo, posto que poderiam oferecer um tipo de sociabilidade, possibilitando uma reconstrução de vida fora do contexto familiar e parental.

Mazo et al12 afirmam ainda, que a influência de amigos e/ou cônjuge gera uma maior probabilidade de indivíduos serem fisicamente ativos, porque os participantes dessa prática, por viverem e sentirem os reais impactos da mesma, tornam-se contribuintes essenciais para a busca dos que ainda não experimentaram, sobretudo quando se trata de pessoas com as quais já têm convivência e confiabilidade.

Não obstante, é interessante destacar a relevância da assistência do enfermeiro da ESF, enquanto cuidador/educador e conhecido da pessoa idosa na contribuição para $o$ envelhecimento ativo e saudável.

\section{Os efeitos da prátioa da Eaminhada em grupo}

As modificações contempladas pela percepção dos sujeitos da pesquisa incluem melhoras de ordem física, mental e social em suas próprias vidas.

\section{Controle das doenças erônieo-degenerativas}

[...] Mudou foi muito, porque ela era sempre alta, sempre era 16, só chegava 16, 18, e agora 14, eu achei que melhorou muito né?

(I1)

[...] O que mudou, foi porque, minha pressão vive controlada, meu diabete. (I3)

[...] achei bom né e aí eu melhorei muito, digo a pressão né? Controlada [...] (I4) 
[...] a pressão está controlada, a diabete está controlada $[. .$.

Melhorou várias coisas, o problema da pressão, a gente vive controlando ela, o diabete, a artrose, que em tudo que a gente faz aquela caminhada, tudo que a gente faz aqueles exercícios, eles vão melhorando a saúde da gente, certo? (risos). (I6)

[...] a pressão normalizou, então [...] tenho até é gozado mais saúde com a aquela caminhada logo bem cedinho (risos). [...] hum! controlou, contanto toda vez que faço a caminhada a pressão volta ao normal e isso é o que eu estou achando bom. (I7)

De acordo com os relatos acima, pode-se inferir que a população estudada percebe a prática da caminhada como mecanismo efetivo no controle do diabetes, artrose e especialmente da hipertensão arterial (HA).

Assim, podemos dizer que os benefícios à saúde ocorrem mesmo quando a prática de atividade física é iniciada em uma fase tardia de vida, por sujeitos sedentários, sendo benéfica inclusive para portadores de doenças crônicas ${ }^{13}$.

Neste caso, relatamos que a prática de atividade física dinamiza a fisiologia corporal de tal maneira que causa uma vasodilatação, diminuindo a tensão nas paredes das artérias e a resistência vascular periférica, culminando com a diminuição da pressão arterial (PA) sistêmica ${ }^{14}$, como podemos verificar através dos relatos dos sujeitos.

Mediante estudos de Farinatti et al15, exercícios com altas intensidades não parecem necessários para que sejam obtidos benefícios na redução da pressão arterial e diminuição nas taxas de morbimortalidade e que exercícios de baixa intensidade seriam tão ou mais eficazes na atenuação da HA quanto aqueles com intensidade elevada.

As informações dispostas pelos pesquisados corroboram com os estudos anteriores, haja vista que o referido controle das patologias é advindo de uma prática de caminhada que se encaixa no perfil de baixa e média intensidade, dependendo da condição clínica e resistência física do idoso.

\section{Redução na massa corpórea}

[...] e olha, eu estava pesando 69 quilo aí eu estava cansada, eu digo, não, eu vou fazer essa caminhada que o povo diz, e agora estou pesando 61 [...] Achei muito, melhorei bastante, melhorei mermo.

(I2)

Entende-se nesse depoimento o contentamento relacionado à redução de peso e a melhora do cansaço. Isso devido seu longo período de prática, conforme evidenciado nos estudos de Caromano et $a^{13}$, onde se percebeu que embora houvesse uma tendência à diminuição do peso e da porcentagem da gordura corpórea com a manutenção dos exercícios, existia a necessidade de um período longo de atividade física para alterar estes parâmetros.

Percebe-se então a importância preventiva de ordem primária e secundária da caminhada, visto que atuaria controlando o armazenamento excessivo de gorduras na região abdominal, que para Farinatti et $a l^{15}$, está relacionada com o desenvolvimento de doenças crônicas cardiovasculares e metabólicas.

Sabe-se que o sobrepeso e a obesidade são condições crescentes em uma abrangência mundial, podendo, dessa forma, serem considerados problemas de saúde pública, e como afirmam Chaim et alli, constituem risco para instalação de incapacidades. Sendo que no indivíduo de idade avançada, adquire dimensão aumentada.

\section{Alivio nos quadros álgieos}

[...] já bastava essa dor em meus braços que eu sentia muito, não estou sentindo mais e assim estou bem melhor. (I2)

[...] tinha dia que eu me sentava assim, eu tinha que botar as pernas em cima de uma cadeira, parece que era aquela dor cansada, e acabou, não sinto mais não. (I3)

[...] e aquele horror de dor que eu tinha também [...] Mudou assim porque eu não saía assim né, aí comecei andar para lá e melhorei muito assim das dores [...]

[...] o que eu sinto muito, muito, muito é essa dor aqui no braço, porque eu tenho um pouquinho de deslocamento aqui, e o médico disse que se eu não melhorasse era para operar [...] com as caminhadas, os exercícios [...] me aliviou demais com as fisioterapia, aquele negócio de jogar os braço aqui, movimenta, eu me sinto muito bem na caminhada[...] (I8) 
Muita coisa, porque eu tinha uma dor muito grande nas pernas e depois da caminhada eu me sinto bem melhor... (I10)

As falas acima revelam a influência da caminhada e dos exercícios fisioterápicos précaminhada, no controle de algias, contribuindo dessa forma para funcionalidade e bem estar da pessoa idosa. $\mathrm{O}$ estresse causado no organismo pela presença de dor é potencializado na pessoa de idade avançada, porque se tem o somatório das alterações e limitações próprias da senescência.

\section{Melhora na saúde mental e social}

[...] aí graças a Deus me ajudou muito esse grupo, porque do jeito que en estava, eu não queria ver ninguém, aí eu me enturmei, parece que me curei, certo? (risos).

[...] tudo é de bom para gente, a gente tira uma coisa que tem assim no sentido, às vezes, a gente está pensando muito em uma coisa, quando se acha no meio daquele horror de gente de idade, a gente conversa, sorrir toda coisa, a gente distrai e aquilo passa, não é? para mim foi muito legal, está bom. (I10)

[...] a gente parece que melhora mermo a vida da gente mais assim, o mau amor (risos), tudo melhorou [...] (I3)

Bom, a gente melhora mais porque aquela solidão que a gente tem, muitas vezes na caminhada junto com as outras pessoas conversando né? Participando e fazendo exercício a gente melhora muito a saúde, né? [...] porque o que adoece o idoso é o isolamento, né? [...] o idoso não pode ser fechado, tem que andar, tem que se comunicar tudo, isso é muito bom, o idoso que só vive em quatro parede ele fica muito mais doente, né? (I8)

A vivência em sociedade é própria do ser humano porque caracteriza sua natureza social, por meio da verbalização feita pelos participes, percebese, que o grupo de caminhada se constitui como promotor de saúde mental, proporcionando possível cura e ressocialização de quem se encontrava em depressão. Este também possibilita a proteção da mente contra os malefícios das numerosas preocupações que ameaçam a pessoa idosa, que, por conseguinte, agora pode conversar e rir em meio a seus semelhantes.

Como documentou Nardi et al17, entre as terapêuticas para depressão está à participação em atividades de grupo. Moraes et al ${ }^{18}$, por meio de revisão de artigos, concluiu que dessas a caminhada e corrida são os tratamentos mais utilizados para níveis graves de depressão, recomendando ainda, atividades longas e menos intensas, argumentando que dessa forma interrompem com maior eficiência os sintomas depressivos.

Tendo em vista que a maioria dos pacientes depressivos é sedentária, preconiza-se a frequência das atividades em duas a quatro vezes por semana, dado que confere com o realizado no grupo objeto do presente estudo ${ }^{18}$.

Percebe-se, através dos depoimentos, a contribuição da caminhada em grupo no tratamento dos sintomas depressivos de quem a pratica. O contato com outras pessoas aumenta o círculo de amizades e, evidentemente, as fontes de ajuda, construindo dessa maneira para o suporte do enfrentamento das dificuldades além do seio familiar.

Os pesquisados retratam ainda, a vivência com as atividades em grupo como colaboradora para a promoção do bem estar. Há um reconhecimento de que os seres humanos são sociais por natureza, condição essa que aflora no período da velhice, como aponta I.8, quando diz que o isolamento, a vida sem sair de casa, é que adoece o idoso.

Verificou-se ainda a presença de sorrisos entremeando as respostas, detalhe interessante e louvável, à medida que comprova o benefício do grupo para o humor de seus integrantes.

Assim, o exercício físico está também associado ao aumento da alegria, da autoeficácia e do autoconceito. Parece que as atividades físicas dão a oportunidade de o indivíduo ter uma sensação de sucesso que, por sua vez, reforça a autoimagem e a autoestima positiva ${ }^{12}$.

\section{Melhora na mobilidade fisiog}

[...] caminhando eu achei mais, desenvolveu mais um pouco né? que a perna que era inchada, que, é essa, que eu caí [...] aí eles diziam assim: olha ela está caminhando melhor! eu digo, vocês acham? Está, no dia que tu veio a 
primeira vez tava toda torta! (risos...)

[...] e aí amoleceu mais os ossos né? (risos) que já era tudo duro, gostei muito e estou gostando, já está com dois anos né? não pretendo deixar não.

(I4)

\section{[...] as pernas da gente desenvolve mais um pouco né? [...] (I9)}

Entende-se a prática da caminhada como promotora e/ou restauradora da elasticidade, flexibilidade e melhoramento postural do idoso.

$\mathrm{O}$ envelhecimento biológico dos humanos, geralmentre, relacionado a diminuição da quantidade de líquidos, da elasticidade dos tecidos e ainda pelo desenvolvimento de patologias nas articulações, sedentarismo e quedas, compromete a manutenção da qualidade na mobilidade física, do equilíbrio e da postura ${ }^{19}$. A caminhada surge então para contornar eficazmente tal situação, pois estimula e mantém a funcionalidade das articulações, pela sucessiva repetição dos movimentos.

Sabe-se que uma boa mobilidade articular tem representativa importância para o sucesso nas atividades cotidianas, sobretudo na prevenção de quedas, causa frequente de internação hospitalar, deterioração da capacidade funcional e até aceleração do processo de finitude entre idosos.

Dado interessante nesse contexto, são os estudos de Farinatti et al ${ }^{15}$ quando demonstram que o tempo de adaptação após treinamento específico é relativamente curto. Fato confirmado pelos resultados de sua pesquisa onde o incremento da flexibilidade de tronco aconteceu de forma rápida. Esses resultados juntamente com os declarados pelos sujeitos pesquisados no presente estudo convergem para a otimização de pensamentos acerca da efetividade da caminhada na promoção do envelhecimento ativo.

\section{Considerações Finais}

O envelhecimento humano é fenômeno merecedor de atenção nesse momento, haja vista, que a inversão da pirâmide demográfica brasileira se manifesta como um grande desafio para a saúde.

$\mathrm{O}$ aumento no número de pessoas atingindo a terceira idade, acompanhado de doenças crônicodegenerativas configuram um quadro preocupante, porque há uma incompatibilidade entre demanda e suporte do sistema de saúde. Porém, a existência de políticas e algumas iniciativas assistenciais já colaboram no enfrentamento dessa realidade. Sendo que a implantação da prática da caminhada de idosos em grupo na Estratégia Saúde da Família representa importante ferramenta.

O presente estudo, realizado por meio de entrevistas a idosos da ESF Mangueira, ao descrever a óptica dos mesmos em relação aos efeitos da vivência dessa estratégia em suas vidas, tornou possível entender que eles percebem-na, como forma de dinamizar positivamente o seu envelhecer. Pois reconhecem e relatam os efeitos benéficos no controle das doenças comuns na idade avançada, na consolidação do bem estar físico, mental e social.

Os resultados encontrados atuam como norte, para a atuação eficaz de todos os membros da equipe de assistência, para a avaliação da efetividade da implementação e desenvolvimento das políticas públicas de saúde em prol dos que envelhecem, bem como no planejamento de novas estratégias que se fizerem necessárias.

Portanto, necessita-se, valorizar os esforços dispensados anteriormente para o aumento da expectativa de vida humana, colocando em prática as políticas públicas existentes em favor da manutenção da funcionalidade e autonomia da pessoa idosa. Só assim, dar-se-á largo passo no caminho que fará a velhice sair da condição de prejuízo para se tornar conquista social.

\section{Referêneias}

1. Mendes MRSSB, Gusmão JL, Faro ACM, Leite RCBO. A situação social do idoso no Brasil: uma breve consideração. Acta Paul Enferm 2005; 18 (4): 422-6.

2. Morais EP, Rodrigues RAP, Gerhardt TE. Os idosos mais velhos no meio rural: realidade de vida e saúde de uma população do interior Gaúcho. Texto Contexto Enferm, Florianópolis, 2008; 17(2):374-83.

3. Brasil, Ministério da saúde. Secretaria de atenção à saúde. Atenção à saúde da pessoa idosa e envelhecimento. Série Pactos pela Saúde 2006, v.12. Brasília (DF) Ministério da saúde; 2010.

4. Benedetti TRB, Gonçalves LHT, Mota JAP da S. Uma proposta de política pública de atividade física para idosos. Texto Contexto Enferm, Florianópolis, 2007; 16(3):387-98.

5. Vono Z E. Enfermagem gerontológica: atenção à pessoa idosa. Série apontamentos. $2^{\mathrm{a}}$ ed. São Paulo: SENAC; 2007.

6. Brasil, Ministério da Saúde. Secretaria de Atenção à Saúde. Revista brasileira: saúde da família. Brasília (DF), Ministério da Saúde; 2006.

7. Goncalves MP, Tomaz C, Sangoi C. Considerações sobre envelhecimento, memória e atividade física. $\mathrm{R}$. Bras. Ci e Mov 2006; 14(2): 95-102.

8. Freitas CMSM, Santiago MS, Viana AT, Leão AC, Freyre C. Aspectos motivacionais que influenciam a adesão e manutenção de idosos a programas de 
exercícios físicos. Rev. Bras. Cineantropom. Desempenho Hum 2007; 9(1): 92-100.

9. Santana RF, Santos I. Como tornar-se idoso: um modelo de cuidar em enfermagem gerontológica. Texto e Contexto Enferm 2005; 14(2):95-102.

10. Brasil, Ministério da saúde. Secretaria de Atenção à Saúde. Caderno de atenção básica $n^{\circ} 19$ : envelhecimento e saúde da pessoa idosa. Brasília (DF); 2007.

11. Bessa MEP, Silva MJ. Motivações para o ingresso dos idosos em instituições de longa permanência e processos adaptativos: um estudo de caso. Texto Contexto Enferm 2008; 17(2):258-65.

12. Mazo GZ, Cardoso FL, Aguiar DL. Programa de Hidroginástica para Idosos: Motivação, Auto-Estima e Auto-Imagem. Rev Bras Cineantropom Desempenho Hum 2006; 8(2):67-72.

13. Caromano FA, Ide MR, Kerbauy RR. Manutenção na prática de exercícios por idosos. Revista do Departamento de Psicologia - UFF, v.18 - n.2 jul./dez; 2006.

14. Santos, Marcos Antônio Pereira dos. O exercício físico na prevenção e tratamento da hipertensão arterial. Teresina: EDUFPI, 2008.
15. Farinatti PTV, Oliveira RB, Pinto VLM, Monteiro WD, Francischetti E. Programa domiciliar de exercícios: efeitos de curto prazo sobre a aptidão física e pressão arterial de indivíduos hipertensos. Arquivos Brasileiros de Cardiologia. v.84 - n.6 jun; 2005.

16. Chaim J, Izzo H, Sera CTN. Cuidar em saúde: satisfação com imagem corporal e autoestima de idosos. O Mundo da Saúde, São Paulo, 2009; 33(2):175-181.

17. Nardi EFR, Miguel MEGB, Stachuka M. Avaliação de estados depressivos em idosos de um grupo de vivência. Revista F@pciência. v. 3, n. 4, p. 41- 52. Paraná, 2009.

18. Moraes H, Deslandes A, Ferreira C, Pompeu FAMS, Ribeiro P, Laks J. O exercício físico no tratamento da depressão em idosos: revisão sistemática. Rev Psiquiatr 2007; 29(1):70-79.

19. Silveira MM, Pasqualotti A, Colussi EL, Wibelinger LM. Envelhecimento humano e as alterações na postura corporal do idoso. Revista Brasileira de Ciência da Saúde, ano 8, n² 26, out/nov; 2010. 